\title{
High resolution imaging of dielectric surfaces with an evanescent field optical microscope
}

\author{
N.F. van Hulst, F.B. Segerink and B. Bölger \\ Opto-electronics, Applied Physics, University of Twente, P.O. Box 217,7500,AE Enschede, Netherlands
}

Received 2 July 1991; revised manuscript received 23 October 1991

\begin{abstract}
An evanescent field optical microscope (EFOM) is presented which employs frustrated total internal reflection on a localized scale by scanning a dielectric tip in close proximity to a sample surface. High resolution images of dielectric gratings and spheres containing both topographic and dielectric information have been obtained. The resolution obtained is $30 \mathrm{~nm}$ in the lateral directions and $0.1 \mathrm{~nm}$ in height depending on proper tip fabrication.
\end{abstract}

\section{Introduction}

Scanning near field optical microscopy (SNOM) is one of the several forms of scanning probe microscopy that have emerged over the last years stimulated by scanning tunneling microscopy (STM) and atomic force microscopy (AFM). In SNOM the near field optical interaction between a dielectric sample surface and an optical antenna of nanometer size is detected. Optical images with a lateral resolution approaching $\lambda / 50$ are obtained with the nanometer size antenna being either a miniature aperture in a metal sheet [1-4] or a sharp dielectric probe tip [5-7]. At variance to STM and AFM the physical active medium in SNOM is the hf electro magnetic field with its specific frequency and polarization. Consequently the image contains both spectroscopic and polarization dependent information. Recently Betzig et al. [8], using a tapered single mode fibre with aiuminum coating, have demonstrated a $12 \mathrm{~nm}$ lateral resolution while the contrast depends on the polarization. Kopelman et al. [9] have produced an ultra-small light source $(\varnothing 50 \mathrm{~nm})$ by growing crystals in a micro-pipette which produce radiatively decaying excitons upon excitation. Fischer and Pohl [10] observed strong field enhancement due to surface plasmon effects using $100 \mathrm{~nm}$ metallized latex spheres as antenna.

We present progress made on our evanescent field optical microscope (EFOM) [7]. In this scheme an evanescent field is generated by total internal reflection (TIR) at a substrate surface. A sample placed in this field causes a spatial variation of the evanescent field which is characteristic for the dielectric and topographic properties of the sample. The TIR is frustrated by a suitably sharpened dielectric probe and the evanescent wave is partly converted into a propagating wave in a fibre and detected. An image is obtained by scanning the probe over the sample surface with nanometer accuracy while keeping the height or the optical flux constant ("optical tunneling"). The lateral resolution is determined by the sharpness of the tip, distance from tip to sample and the evanescent field gradient.

A similar set-up, referred to as photon scanning tunneling microscope (PSTM), has been reported by Reddick et al. [5] and Courjon et al. [6]. The PSTM has been demonstrated in a spectroscopic mode [11] and applied to waveguides [12]. Although $5 \mathrm{~nm}$ lateral resolution has been claimed [6] caution should be taken in the interpretation of these images because of polarization effects and tip artifacts. Moreover radiative scattering plays a substantial role, as was shown [13].

The principle of operation of the EFOM is discussed in further detail and images of dielectric surfaces with a lateral resolution down to $30 \mathrm{~nm}$ are presented and discussed. 


\section{Principle of operation}

TIR is a familiar phenomenon which occurs when light reflects at an interface from an optically dense medium to a rarer medium with refractive indices $n_{1}$ and $n_{2}\left(<n_{1}\right)$ respectively, at an angle of incidence $\theta$ larger than the critical angle $\theta_{c}=\arcsin \left(n_{2} / n_{1}\right)$. In the denser medium a standing wave normal to the interface is established, whereas in the rarer medium there is a nonpropagating exponentially decaying field $E(z)=E(0) \exp \left(-z / d_{\mathrm{p}}\right)$ as drawn in fig. 1a. Herein $z$ is normal to the interface and $d_{\mathrm{p}}$, the penetration

(a)

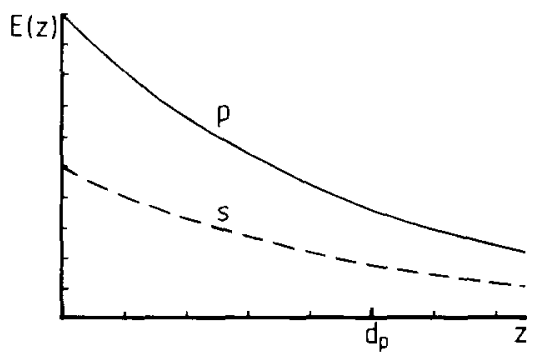

(b)
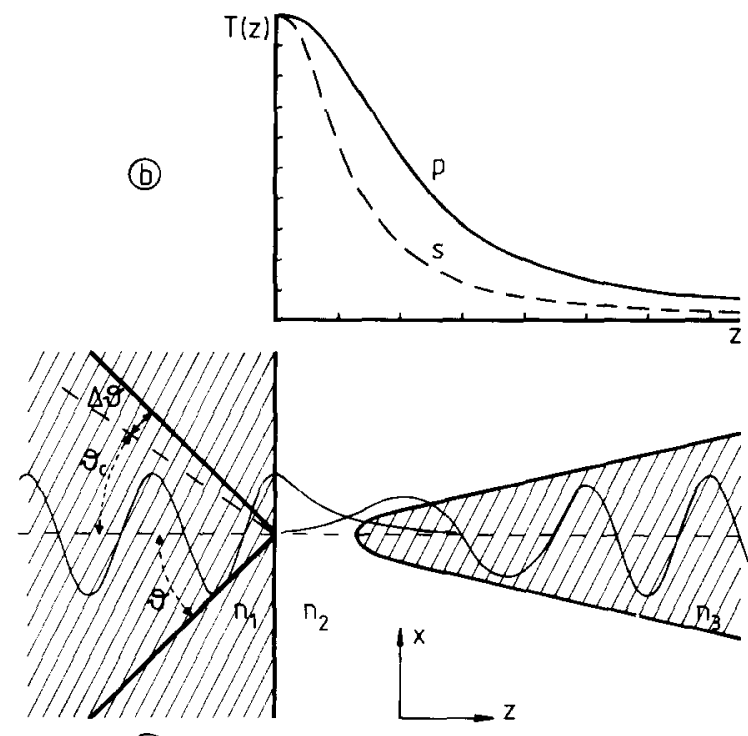

(C)

Fig. 1. (a) Decay of the evanescent field $E(z)$ in the rarer medium caused by TIR for $\mathrm{p}$ and s polarization. (b) Optical transmission $T(z)$ caused by frustrated total internal reflection between two dielectric media with separation $z$. (c) Localized FTR by a sharp dielectric tip, schematically showing the overlap of the evanescent fields between the planar interface and the probe, with refractive indices $n_{1}$ and $n_{3}$, respectively. depth of the evanescent field into the rarer medium, is given by

$d_{\mathrm{p}}=(\lambda / 2 \pi)\left(n_{1}^{2} \sin ^{2} \theta-n_{2}^{2}\right)^{-1 / 2}$

and is typically one-tenth the wavelength $(\lambda)$ for grazing incidence and increases to several wavelengths for incidence close to $\theta_{c}$. The field components $E_{x}$ and $E_{y}$ parallel to the surface are continuous at the interface, while the normal component $E_{\text {. }}$ is discontinuous, being larger by a factor $\left(n_{1} / n_{2}\right)^{2}$ in the rarer medium due to the continuity of the displacement $D_{z}$. Hence the evanescent field magnitude is larger for p-polarization than for s-polarization by a factor depending on the refractive index.

If a third dielectric medium is brought within a few penetration depths of the interface the total reflection is reduced, called frustrated total reflection (FTR), and part of the light is transmitted into the third medium. The fraction of the transmitted intensity $T(z)$ between two semi-infinite media is given by [14]

$$
T(z)^{-1}=1+\frac{\sinh ^{2}\left(z / d_{\mathrm{p}}\right)}{\sin ^{2}(\delta)},
$$

where $\delta$ is the phase shift upon reflection and $z$ the separation distance. The transmission decays exponentially proportional to $\exp \left(-2 z / d_{\mathrm{p}}\right)$ for large $z$, while it levels off towards $100 \%$ at small separation $z \ll d_{\mathrm{p}}$ (fig. $1 \mathrm{~b}$ ).

Eq. (2) is not valid for FTR by a dielectric tip with a radius smaller than $\lambda$. For this case the overlap of the evanescent fields at the planar interface and the tapered probe has to be calculated (fig. 1c). The presence of disturbance or a sample results in a combination of evanescent and radiating fields. Labani [15] and Girard [16,17] have presented theoretical models which describe the interaction between a light scattering micro-particle and the evanescent field at a dielectric surface. Also surface corrugation has been taken into account by calculating localized multipolar interactions. The exponential behaviour of the optical coupling efficiency causes a field sharpening effect additional to the tip sharpness. Consequently the lateral resolution is better than expected from the tip size. This is analogous to the situation in STM and AFM where atomic resolution is obtained due to field sharpening. 


\section{Apparatus}

The evanescent field is generated by TIR of a linearly polarized $\mathrm{HeNe}$ laser beam in a glass substrate (Schott BK7, $n=1.515$ ). The probe tip is fabricated from a quartz fibre sharpened to about $100 \mathrm{~nm}$ radius with an apex of $\sim 20^{\circ}$, by chemical etching at the meniscus between a $20-40 \% \mathrm{HF}$ solution and paraffin oil. The fraction of the laser power coupled into the optical probe by FTR is typically $10^{-7}$. This fraction is mainly determined by the ratio between the probe tip area $(\sim \varnothing 100 \mathrm{~nm})$ and the waist $(\sim \varnothing 100 \mu \mathrm{m})$ of the laser beam focus. Taking this into account typically $10 \%$ of the light power present at the probe surface is coupled in, while $100 \%$ is expected for contact.

An image is obtained by manipulating the optical probe tip in lateral and vertical directions over the sample surface by means of a piezo-electric actuator. The scanning action is performed either in constant height mode, resulting in an image presenting the optical signal variation, or in constant optical flux mode, resulting in an image representing topography and variation in dielectric constant, which is obtained by a feedback on the vertical piezo element.

\section{Microscopic images}

The optical coupling between a clean glass (BK7) substrate and the probe tip as a function of the separation $z$ the angle $\Delta \theta=\theta-\theta_{\mathrm{c}}$ and the polarization direction has been investigated [7]. An effective decay length was fitted to the measured exponential decay of the coupled signal at large $z$, which is plotted in fig. 2 as a function of $\Delta \theta$, together with the theoretical value of $d_{\mathrm{p}} / 2$ for an undisturbed evanescent field decay, as expected from eq. (2). The correspondence is reasonable, also for other tips used. A similar correspondence was observed by Reddick [5]. Slight deviations can be attributed to an uncertainty in $\Delta \theta$ and to laser beam divergence $\left(0.1^{\circ}\right)$.

The presence of a sample introduces a change in the evanescent field distribution. However the sample surface roughness also results in a non-evanescent field, which extends beyond the penetration depth of the evanescent field and thus disturbs the optical feedback action. Therefore, depending on the

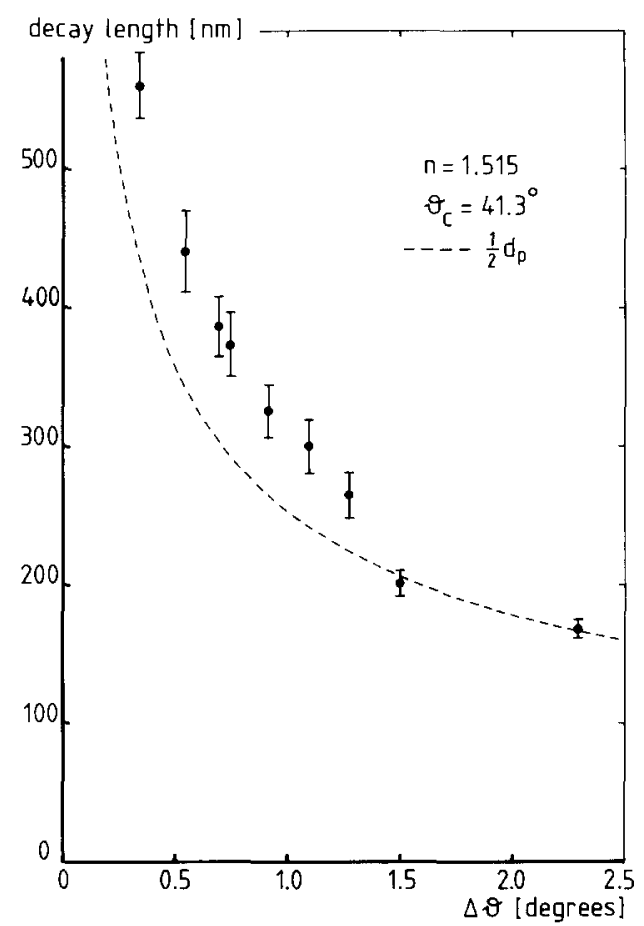

Fig. 2. Experimentally determined effective decay length as a function of the angle $\Delta \theta=\theta-\theta_{\mathrm{c}}$ for $n=1.515$ and the theoretical value of $d_{\mathrm{p}} / 2$ (dashed curve).

type of sample which determines the ratio between the evanescent and radiating field contribution, a suitable detection mode has to be chosen.

We have examined dielectric gratings and latex spheres prepared on a microscope cover slip and placed on the glass subtrate together with an immersion liquid.

Fig. 3 shows EFOM images of a silicon-nitride grating. The grating has been fabricated by lithographic etching, using a $1 \mu \mathrm{m}$ period mask, of a $\mathrm{Si}_{3} \mathrm{~N}_{4}$ layer obtained by pressure enhanced chemical vapour deposition. The image of a $3 \mu \mathrm{m} \times 3 \mu \mathrm{m}$ area with steps $500 \mathrm{~nm}$ wide and $500 \mathrm{~nm}$ high, illuminated by TIR with a s-polarized beam and scanned in constant flux mode is plotted in fig. 3a. A similar image of steps $500 \mathrm{~nm}$ wide and $350 \mathrm{~nm}$ high, scanned with a sharper tip is plotted in fig. $3 \mathrm{~b}$. Both images are plotted in an emission contour without further filtering or smoothing of the data and display the topographic structure associated to a surface of constant optical coupling. In fig. 3a the trenches are 

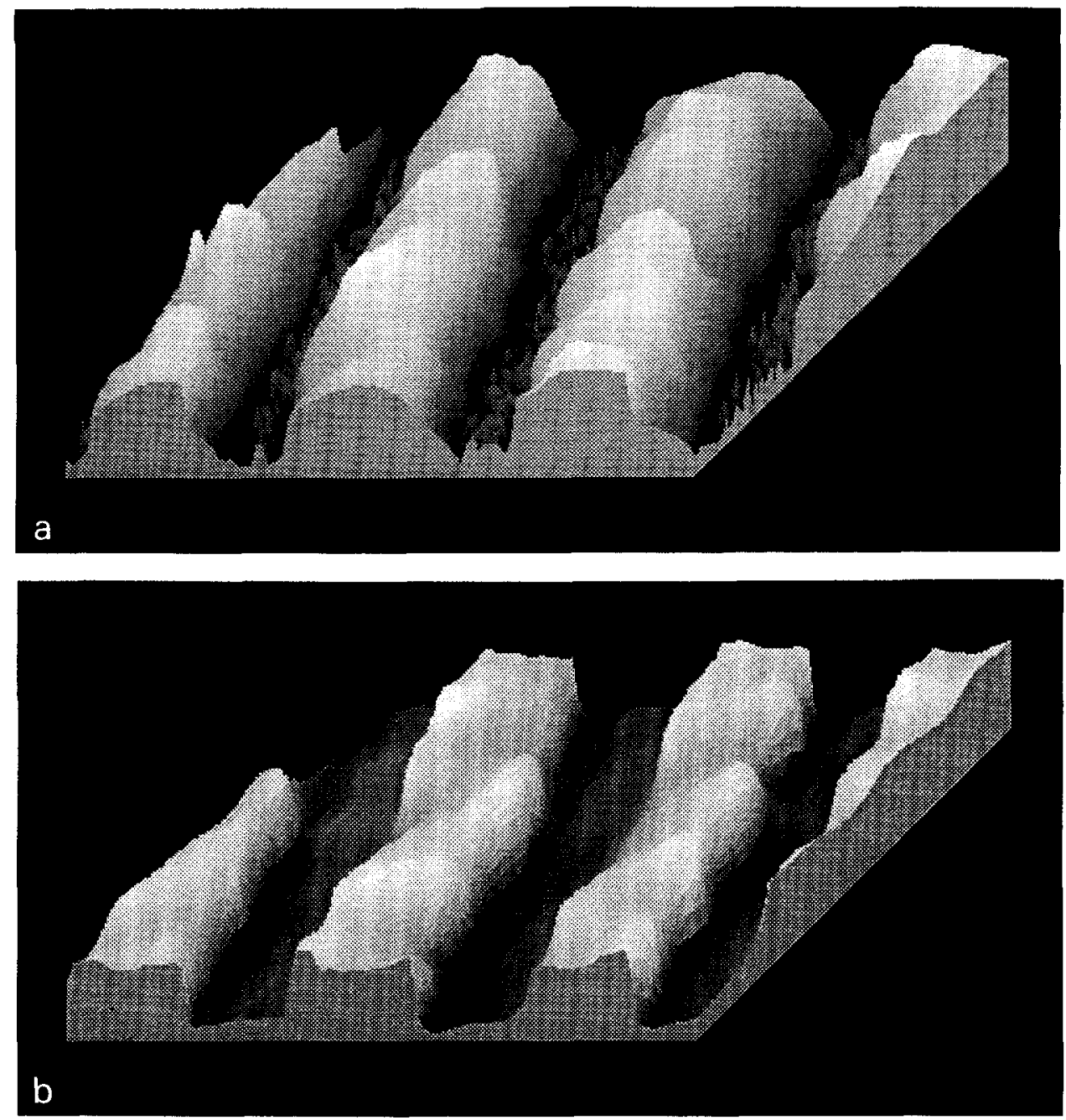

Fig. 3. (a) Emission contour image of a $3 \mu \mathrm{m} \times 3 \mu \mathrm{m}$ area of a SiN grating with steps $500 \mathrm{~nm}$ wide and $500 \mathrm{~nm}$ high in constant flux mode. (b) As (a), with a sharper tip and steps $350 \mathrm{~nm}$ in height.

deep so that the tip can barely couple to the bottom region, resulting in a noisy profile caused by optical coupling of the side of the tip to the grating edges. In fig. $3 b$ the trenches are less deep and the tip is so sharp that it follows the grating contour with an average edge width of $30 \mathrm{~nm}$. Although the actual grating is flat on the top, as verified with an AFM, the top face of the grating steps appears smoothly modulated in the EFOM images. This modulation reflects the presence of interfering scattered waves above the grating.
An EFOM image of latex spheres is shown in fig. 4. The sample was prepared by evaporation of a solution of $\varnothing 951 \mathrm{~nm}$ latex spheres in water and methanol. An EFOM image, in constant flux mode, of a $20 \mu \mathrm{m} \times 20 \mu \mathrm{m}$ area with a few latex spheres is displayed in fig. 4a. The spheres can be clearly distinguished and the detected peak height is $1.4 \mu \mathrm{m}$ above the surface. Fig. $4 \mathrm{~b}$ is a scan of the same area, but one of the latex spheres was moved to the edge of the scan region by the scanning tip, while other features are well reproduced. To each sphere a ridge pattern 

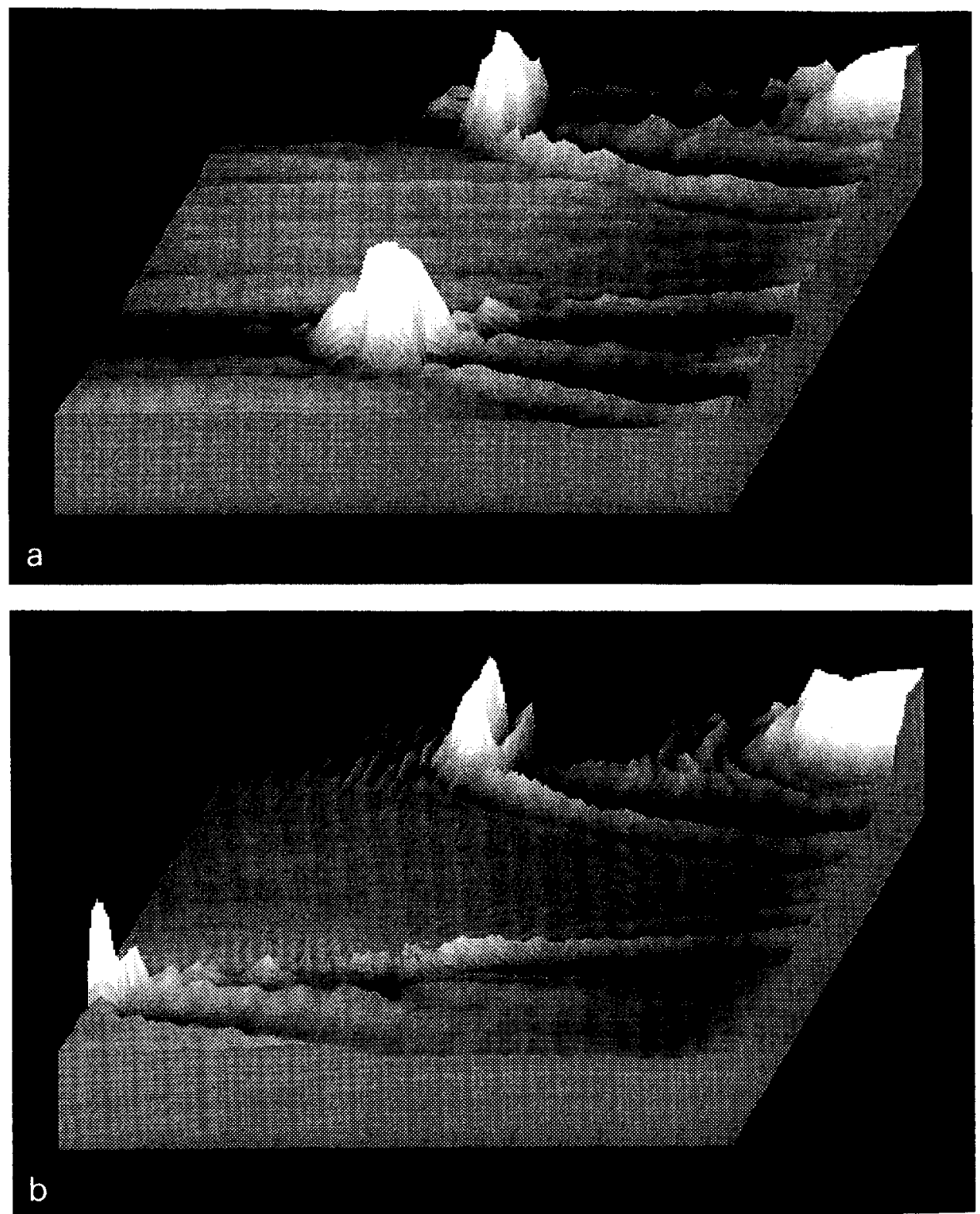

Fig. 4. (a) $20 \mu \mathrm{m} \times 20 \mu \mathrm{m}$ image of $\varnothing 951 \mathrm{~nm}$ latex spheres with laser beam incident from left to right. (b) Similar to fig. 3a, with one latex sphere moved by the tip to the edge of the scan region.

is associated with a maximum height of about $90 \mathrm{~nm}$. The direction of the ridges is determined by the direction of the incident laser beam, propagating from left to right in figs. $4 \mathrm{a}$ and $4 \mathrm{~b}$. They are not related to topographic features but are interference phenomena. Both the evanescent wave, which propagates as a planar wave along the surface, and the scattered waves of each sphere are probed by the tip and their mutual interference on the detector affects the detected optical coupling. In constant flux mode the feedback adjusts the vertical piezo element according to the interference pattern which results in the 


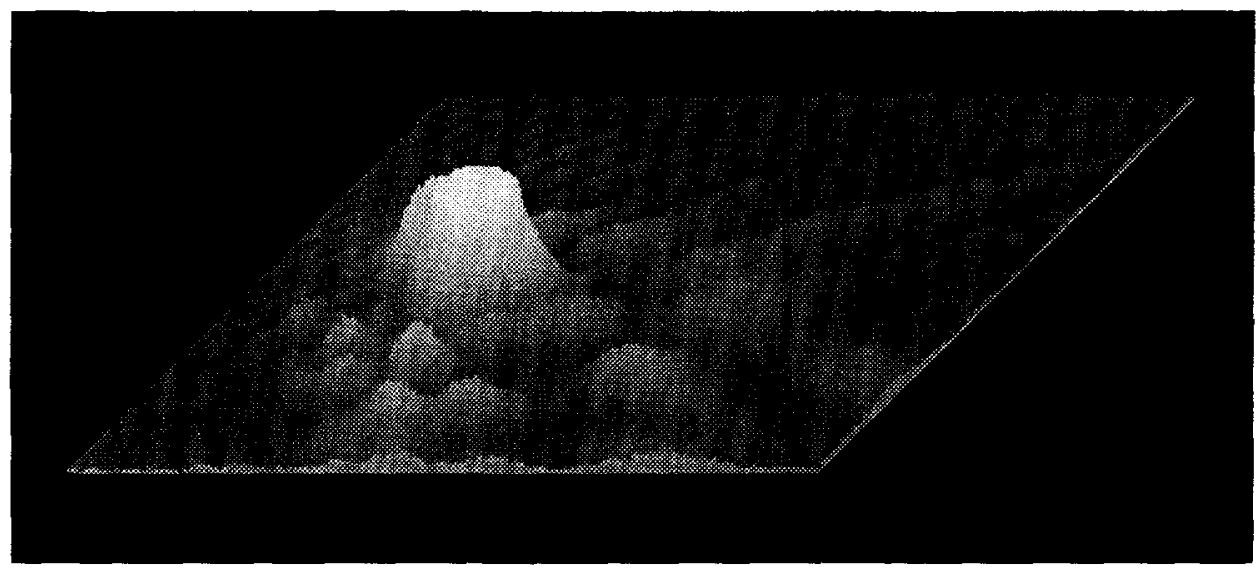

Fig. $5.8 \mu \mathrm{m} \times 8 \mu \mathrm{m}$ image of a $\varnothing 91 \mathrm{~nm}$ latex sphere, scanned constant height mode, displaying the variation in optical coupling.

observed ridges. Similar features have been observed by Reddick [5] at a surface containing $\varnothing 481 \mathrm{~nm}$ wells. These intriguing features will be the subject of a subsequent publication.

Fig. 5 displays the near field distribution of a $\varnothing 91$ $\mathrm{nm}$ latex sphere, scanned over a $8 \mu \mathrm{m} \times 8 \mu \mathrm{m}$ area in constant height mode. The laser beam is incident from left to right. The size of the sphere is determined by radiative field effects which dominate the evanescent field close to the sphere. The diffraction pattern is weak due to the small cross-section of the sphere and because of the constant height scanning mode for which resolution decreases exponentially with distance.

\section{Conclusions}

The observed decay length of the optical coupling corresponds quantitatively to the theoretical characteristics for frustrated total internal reflection.

Our results clearly demonstrate the capacity of the EFOM to obtain sub-wavelength resolution. The lateral resolution of $\sim 30 \mathrm{~nm}$ is determined by the sharpness of the probe tip, the exponential decay of the evanescent field, working distance and the type of sample surface. The vertical resolution is about $0.1 \mathrm{~nm}$, limited by piezo hysteresis and electronic noise.

EFOM images display variation in topography and dielectric properties with sub-wavelength resolution.
Generally the images correspond to the expected structure of the sample but for complete understanding they have to be confronted with a more quantitative theory.

The presence of radiative waves over the sample surface is an important drawback as it distorts the obtained image and may obstruct the feedback action in constant flux mode. This is a problem in the application of the EFOM, which could partially be eliminated by $z$-modulation or a substrate with higher refractive index.

Near field optical microscopy is unique among the scanning probe microscopies as it probes dielectric properties, as such it is a useful extension to STM and AFM when applied to chemical and biological surfaces, especially when its spectroscopic capabilities are exploited.

\section{Acknowledgements}

We kindly thank R.G. Heideman and H. de Vries for supplying the peCVD $\mathrm{Si}_{3} \mathrm{~N}_{4}$ gratings.

\section{References}

[1] U. Dürig, D.W. Pohl and F. Rohner, J. Appl. Phys. 59 (1986) 3318.

[2] E. Betzig, A. Lewis, A. Harootunian, M. Isaacson and E. Kratschmer, Biophys. J. 49 (1986) 269. 
[3] E. Betzig, M. Isaacson and A. Lewis, Appl. Phys. Lett. 51 (1987) 2088.

[4] U.Ch. Fischer, U.T. Dürig and D.W. Pohl, Appl. Phys. Lett. 52 (1988) 249.

[5] R.C. Reddick, R.J. Warmack, D.W. Chilcott, S.L. Sharp and T.L. Ferrell, Rev. Sci. Instrum. 61 (1990) 3669.

[6] D. Courjon, J.-M. Vigoureux, M. Spajer, K. Sarayeddine and S. Leblanc, Appl. Optics 29 (1990) 3734.

[7] N.F. van Hulst, N.P. de Boer and B. Bölger, J. Microsc. 163 (1991) 117 .

[8] E. Betzig, J.K. Trautman, T.D. Harris, J.S. Weiner and R.L. Kostelak, Science 251 (1991) 1468.

[9] R. Kopelman, A. Lewis and K. Lieberman, J. Luminesc. 45 (1990) 298.

[10] U.Ch. Fischer and D.W. Pohl, Phys. Rev. Lett. 62 (1989) 458 .
[11] M.A. Paesler, P.J. Moyer, C.J. Jahncke, C.E. Johnson, R.C. Reddick, R.J. Warmack and T.L. Ferrell, Phys. Rev. B 42 (1990) 6750 .

[12] D.P. Tsai, H.E. Jackson, R.C. Reddick, S.H. Sharp and R.J. Warmack, Appl. Phys. Lett. 56 (1990) 1515.

[13] N.F. van Hulst, F.B. Segerink and B. Bölger, An evanescent field optical microscope; scanning probe microscopy: STM \& beyond, ed. H.K. Wickramasinghe, Series of the American Institute of Physics (1991).

[14] S. Zhu, A.W. Yu, D. Hawley and R. Roy, Am. J. Phys. 54 (1986) 601.

[15] B. Labani, C. Girard, D. Courjon and D. Van Labeke. J. Opt. Soc. Am. B 7 (1990) 936.

[16] C. Girard and M. Spajer, Appl. Optics 29 (1990) 3726.

[17] C. Girard and D. Courjon, Phys. Rev. B 42 (1990) 9340. 